\title{
Gaussian Mixture Unscented Particle Filter with Adaptive Residual Resample for Nonlinear Model
}

\author{
Na Zhang ${ }^{\mathrm{a}}$, Xinxin Yang \\ College of Electronic Information Engineering, Beihang University, Beijing, China \\ anazhang_bhu@163.com, byangxx@buaa.edu.cn
}

\begin{abstract}
To solve nonlinear non-Gaussian filter problems in target tracking, Gaussian mixture unscented particle filter with adaptive residual resample algorithm is proposed. Gaussian mixture unscented particle filter is utilized as importance density to improve the estimation accuracy evidently. By introducing adaptive residual resample, the new algorithm overcomes the defects of general resample algorithm. To evaluate the proposed algorithm, the random surfer dynamic model and range-rate measurement are involved as nonlinear models with two static sensors. Simulation results show that the proposed algorithm performs robust and effective. As a consequence, compared with the general Gaussian particle filter, the proposed algorithm is more accurate in estimated state and more diverse in particles.
\end{abstract}

Keywords-Target tracking; Gaussian mixture; Unscented particle filter; Residual resample

\section{INTRODUCTION}

In the last decades, particle filters (PF) have been popularly used in many fields such as image processing, target tracking, computer vision, economics and management. Particle filters, utilizing the Sequential Monte Carlo (SMC) approach to approximate the Bayesian estimation [1], which are a type of powerful tool to dealing with the nonlinear nonGaussian filtering problems. Their main mechanism is to propagate a set of weighted samples, or particles, and the estimated state can be calculated with these weighted particles. The particles are updated by implementing sequential importance sampling (SIS) process recursively as new measurement information becomes available [2].

Initial particles are sampled from prior probability density function and particle weights are assigned by importance density. As a result, the particle filters efficiency depends on the choice of importance density. The principle of importance density is to minimize the state variance. Optimal importance density is a conditional posterior density of the state. However, it is difficult to sample particles from the posterior density. Instead, importance density is used to approximate it, and it is common to choose Gaussian density [5]. For many medium nonlinear models single Gaussian density has proven effective, however, for deep nonlinear and higher dimensionality models, the performance becomes bad.

In this paper, a new algorithm for importance density in particle filters is proposed. The unscented particle filter with Gaussian mixture algorithm is introduced as the importance density, in which more measurement information is used.
Gaussian mixture method is used in unscented particle filter to improve the performance, we name this algorithm the Gaussian mixture unscented particle filter (GM-UPF).

However, a common problem with SIS particle filter is that the particle importance weights increase stochastically over time, thereby most particle weights are almost zero, which called particle degeneracy. To solve the problem, sampling Importance resampling (SIR) is developed. Various resample methods have been developed in recent years, including multi-nominal resample, residual resample, stratified resample and systematic resample [6]. Unfortunately, resampling causes sample impoverishment problem, which results in loss of diversity of particles.

To circumvent sample impoverishment, adaptive residual resampling is proposed, which avoids uncensored replacing lower weight particles with higher weighted particles and has the ability to maintain sufficient diversity of particles without increasing the particles size.

The rest of the paper is organized as follows. In section II , system model is introduced in detail. Section III presents the principle and realization of the proposed GM-UPF and adaptive residual resample. Simulations results are presented in section IV. Finally, conclusions are given in section $\mathrm{V}$.

\section{SYSTEM MODELS}

\section{A. Model Description}

Consider the problem of target tracking for the following nonlinear discrete-time system. The state space model and measurement model [2] are represented by:

State model

$$
s_{k}=f_{k}\left(s_{k-1}, w_{k-1}\right)
$$

Measurement model

$$
z_{k}=h_{k}\left(s_{k}, v_{k}\right)
$$

Where $f_{k}: R^{n} \times R^{r} \rightarrow R^{n}$ is a nonlinear function as the state transition function to calculate state vector $s_{k} \in R^{n}$, and $w_{k-1} \in R^{r}$ is an independent identically distributed (i.i.d) process noise vector whose known distribution is independent of time. At each discrete time point $k$, 
$z_{k} \in R^{m}$ denotes the system measurement output, according to the state vector $S_{k}$ and measurement model as shown in Eq. (2). $h_{k}: R^{n} \times R^{p} \rightarrow R^{m}$ is the system measurement function, and $v_{k} \in R^{p}$ is another i.i.d measurement noise vector.

\section{B. State Model}

In order to evaluate the performance of the proposed nonlinear algorithm, we consider the random surfer dynamic model [14]. The dynamic state model in Eq. (1) is

$$
S_{k+1}=\left[\begin{array}{cccc}
1 & 0 & \Delta t & 0 \\
0 & 1 & 0 & \Delta t \\
0 & 0 & 1 & 0 \\
0 & 0 & 0 & 1
\end{array}\right] S_{k}+\left[\begin{array}{cc}
\frac{\Delta t^{2}}{2} & 0 \\
0 & \frac{\Delta t^{2}}{2} \\
\Delta t & 0 \\
0 & \Delta t
\end{array}\right]\left[\begin{array}{l}
w_{k}^{x} \\
w_{k}^{y}
\end{array}\right]
$$

Where $\Delta t$ is sample interval, $w_{k}^{x}$ and $w_{k}^{y}$ are zero-mean uncorrelated Gaussian white accelerate changing with time.

The dynamic state vector $S_{k}$ in the Eq. (3) is described as follows:

$$
s_{k}=\left[x, y, v_{x}, v_{y}\right]_{k}^{T}
$$

$(x, y)$ is coordinate of the target, $v_{x}$ is velocity of the $\mathrm{x}$ direction, while $v_{y}$ is velocity of the y direction.

\section{Measurement Model}

For tracking the coordinate and the velocity of a moving target, range and range rate are used as the measurement vector. Range, between the target and sensor, is given by:

$$
R=\left[\left(x_{v}-x_{o}\right)^{2}+\left(y_{v}-y_{o}\right)^{2}\right]^{1 / 2}
$$

Where $\left(x_{o}, y_{o}\right)$ is sensor coordinate and it is fixed, $\left(x_{v}, y_{v}\right)$ is target coordinate and it is changing with time. $\mathrm{R}$ is always positive according the definition.

Range rate, also called the Doppler or radial velocity, is the velocity along a line extending from the sensor to the target.

Range rate can be derived from range [9]. Range rate $(\dot{R})$ is given by $d R / d t$

$$
\begin{aligned}
\dot{R}=\frac{d R}{d t} & =\frac{2\left[\left(x_{v}-x_{o}\right) v_{v}^{x}+\left(y_{v}-y_{o}\right) v_{v}^{y}\right]}{2\left[\left(x_{v}-x_{o}\right)^{2}+\left(y_{v}-y_{o}\right)^{2}\right]^{1 / 2}} \\
& =\frac{\left[\left(x_{v}-x_{o}\right) v_{v}^{x}+\left(y_{v}-y_{o}\right) v_{v}^{y}\right]}{R}
\end{aligned}
$$

Where $v_{v}^{x}$ and $v_{v}^{y}$ are velocity of target and given by state vector in section $B$.

For testing purposes, two static sensors are used in measurement model. The measurement model is defined as follows:

$$
z_{k}=\left[R_{a}, \dot{R}_{a}, R_{b}, \dot{R}_{b}\right]_{k}
$$

Where $R_{a}$ and $R_{b}$ are the range between sensor a (or b) and target. $\dot{R}_{a}$ and $\dot{R}_{b}$ are range rate to sensors.

\section{GAUSSIAN MIXTURE UNSCENTED PARTICLE FILTER WITH ADAPTIVE RESIDUAL RESAMPLE}

\section{A. Gaussian Mixture Unscented Particle Filter}

The Gaussian mixture unscented particle filters employ the unscented transformation to generate more suitable posterior PDF and use mixture Gaussian approximation to produce more accurate estimated state. The advantage of GM-UPF is that it can handle the heavy-tailed distributions better.

We assume the state vector is $s_{k}$ which owns $n$ elements. Its mean is $\bar{s}$ and variance is $P_{s}$. The basic idea of the unscented particle filter is that choosing $2 n+1$ weighted samples $S=\left\{\chi_{j}, w_{j}\right\}$ to approximate the random variable distribution of $s$ according to the $\bar{s}$ and $P_{s}$. We called these weighted samples as $\chi$ point.

Here the definition of the $\chi$ point for each particle $i$, and for each time $k$ is shown as follows,

$$
\chi_{k}^{(i) j}= \begin{cases}\bar{s}_{k}^{i} & j=0 \\ \bar{s}_{k}^{i}+\left(\sqrt{(n+\lambda) P_{s}}\right)_{j}, & j=1, \cdots n \\ \bar{s}_{k}^{i}-\left(\sqrt{(n+\lambda) P_{s}}\right)_{j}, & j=n+1, \cdots, 2 n\end{cases}
$$

For each sigma point ${ }^{j}$, its related weight is

$$
w^{j}=\left\{\begin{array}{l}
w_{m}^{0}=\lambda /(n+\lambda) \\
w_{c}^{0}=\lambda /(n+\lambda)+\left(1-\alpha^{2}+\beta\right) \\
w_{m}^{j}=w_{c}^{j}=1 /[2(n+\lambda)], j=1, \cdots, 2 n
\end{array}\right.
$$

Where $\lambda=\alpha^{2}(n+\kappa)-n, w_{m}^{j}$ represents the mean weight of sigma point, while $w_{c}^{j}$ is the covariance weight. $\alpha, \beta$ and $\kappa$ are parameters of sigma point, and they can be determined by experiments.

For each particle $i$, the state vector of particles is updated using unscented transformation and Gaussian 
approximation, and for each time $k$, we make the following steps.

Initialization:

Initializing the particles to get mean $\bar{S}_{0}^{i}$ and covariance

$P_{s}$ according to Gaussian density.

Calculate sigma points:

Use Eq. (20) to obtain sigma points $\chi_{k-1}^{(i) j}$ at time $\mathrm{k}(\mathrm{k}>0)$. Update time:

$$
\begin{gathered}
\chi_{k \mid k-1}^{(i) j}=f\left(\chi_{k-1}^{(i) j}\right) \\
\bar{S}_{k \mid k-1}^{(i)}=\sum_{j=0}^{2 n} w_{m}^{j} \chi_{k \mid k-1}^{(i) j} \\
P_{\mathrm{s}, k \mid k-1}^{(i)}=\sum_{j=0}^{2 n} w_{c}^{j}\left[\chi_{k \mid k-1}^{(i) j}-\bar{s}_{k \mid k-1}^{(i)}\right]\left[\chi_{k \mid k-1}^{(i) j}-\bar{S}_{k \mid k-1}^{(i)}\right]^{T}+Q_{k} \\
\gamma_{k \mid k-1}^{(i) j}=h\left(\chi_{k-1}^{(i) j}\right) \\
\bar{Z}_{k \mid k-1}^{(i)}=\sum_{j=0}^{2 n} w_{m}^{j} \gamma_{k \mid k-1}^{(i) j}
\end{gathered}
$$

Update measurement:

$$
\begin{gathered}
P_{z, k \mid k-1}^{(i)}=\sum_{j=0}^{2 n} w_{c}^{j}\left[\gamma_{k \mid k-1}^{(i) j}-\bar{z}_{k \mid k-1}^{(i)}\right]\left[\gamma_{k \mid k-1}^{(i) j}-\bar{Z}_{k \mid k-1}^{(i)}\right]^{T}+R_{k} \\
P_{s z, k}^{(i)}=\sum_{i=0}^{2 n} w_{c}^{j}\left[\chi_{k \mid k-1}^{(i) j}-\bar{S}_{k \mid k-1}^{(i)}\right]\left[\gamma_{k \mid k-1}^{(i) j}-\bar{Z}_{k \mid k-1}^{(i)}\right]^{T} \\
K_{k}=P_{s z, k}^{(i)}\left(P_{z, k \mid k-1}^{(i)}\right)^{-1} \\
\bar{S}_{k}^{(i)}=\bar{S}_{k \mid k-1}^{(i)}+K_{k}\left(z_{k}-\bar{Z}_{k \mid k-1}^{(i)}\right) \\
P_{s, k}^{(i)}=P_{s, k \mid k-1}^{(i)}-K_{k} P_{z, k}^{(i)} K_{k}^{T}
\end{gathered}
$$

Evaluate the weight and normalize:

Gaussian mixture method is applied in evaluating the particle weight, one Gaussian approximation use prior component message with unscented transformation, another one Gaussian approximation use measurement information.

$$
\begin{gathered}
\bar{z}_{k}^{(i)}=f\left(\bar{s}_{k}^{(i)}\right) \\
w_{1, k}^{i}=e^{-\frac{1}{2}\left(z_{k}-\bar{z}_{k}^{(i)}\right)^{T} \operatorname{inv}(R)\left(z_{k}-\bar{z}_{k}^{(i)}\right)} \\
w_{2, k}^{i}=e^{-\frac{1}{2}\left(\bar{s}_{k}^{(i)}-s_{k}\right)^{T} \operatorname{inv}(Q)\left(\bar{s}_{k}^{(i)}-s_{k}\right)}
\end{gathered}
$$

$$
w_{k}^{i}=w_{1, k}^{i}+w_{2, k}^{i}
$$

We get the estimated state

$$
\tilde{s}_{k}=\sum_{i=1}^{N} w_{k}^{i} \bar{s}_{k}^{i}
$$

\section{B. Adaptive Residual Resample}

A common problem in particle filters is particle degeneracy phenomenon in which a number of particles are devoted to update particles whose contribution to approximation of $p\left(z_{k} \mid s_{k}^{i}\right)$ is almost zero. To solve the degeneracy phenomenon, resampling is introduced. The basic idea of resampling is to eliminate particles that have small weights and to concentrate on particles with large weights. However, over resampling quickly results in impoverishment problem which leads to lose of diversity in particles.

In this section, adaptive residual resample method is proposed which is simple but effective and compensates some support particles to improve residual resampling process. The steps of adaptive residual resample are:

(1) For the particles $\left\{s_{k}^{i}, w_{k}^{i}\right\}_{i=1}^{N}$, all weights are multiplied by the ensemble size $\mathrm{N}$. Then $n_{k}^{i}$ copy particles are taken of each particle $s_{k}^{i}$ in which $n_{k}^{i}=\left\lfloor N w_{k}^{i}\right\rfloor$. The whole number of copy particles is $N^{\prime}=\sum_{i=1}^{N} n_{k}^{i}$, therefore we obtain the new particles $\left\{s_{k}^{i}, w_{k}^{i}\right\}_{i=1}^{N^{\prime}}$.

Calculate the residual particles $m=N-N^{\prime}$. If $m>0$, go to step (2), otherwise, go to step (3).

(2) Allocating new weights for all the particle:

$$
w_{k}^{i^{\prime}}=\left(N w_{k}^{i}-n_{k}^{i}\right) / m
$$

The new distributed particles $\left\{s_{k}^{i^{\prime}}, w_{k}^{i^{\prime}}\right\}_{i=1}^{N}$ is generated, in which we choose the m max weighted particles as spread copy particles.

$$
\left\{s_{k}^{i}, w_{k}^{i}\right\}_{i^{\prime}=1}^{m}=\max _{m}\left\{w_{k}^{i^{\prime}}, s_{k}^{i^{\prime}}\right\}_{i=1}^{N}
$$

(3) Draw the residual resample particles:

$$
\left\{\tilde{s}_{k}^{i}\right\}_{i^{\prime}=1}^{N}=\left\{\left\{s_{k}^{i}\right\}_{i=1}^{N^{\prime}},\left\{s_{k}^{i}\right\}_{i^{\prime}=1}^{m}\right\}
$$




\section{Gaussian Mixture Unscented Particle Filter with Adaptive Residual Resample Algorithm}

We propose the algorithm combining Gaussian mixture unscented particle filter with adaptive residual resample to improve particle filter propagation.
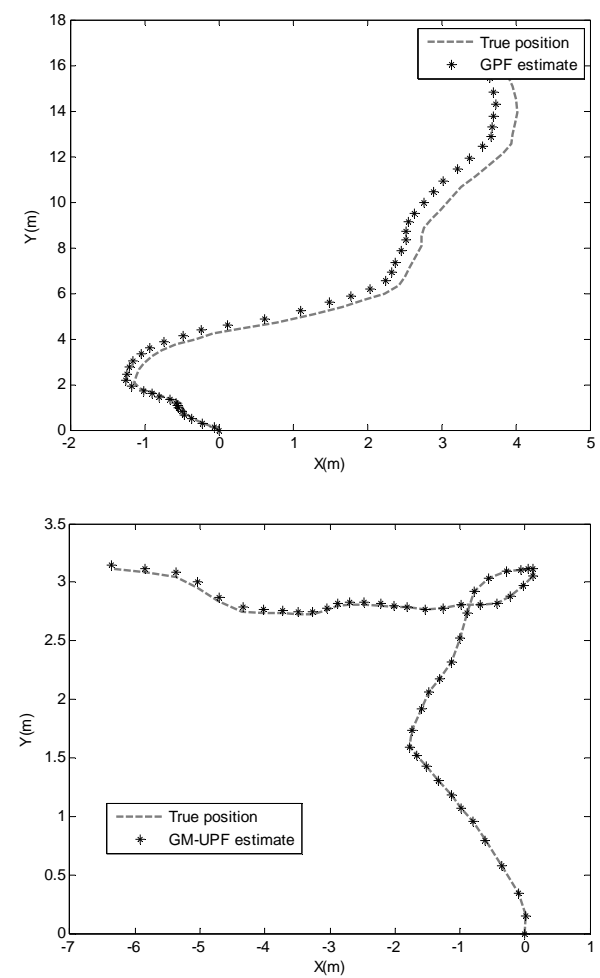

Figure 1. True position and the estimates of GPF and GM-UPF

The steps of the Gaussian mixture unscented particle filter with adaptive residual resample algorithm are shown as follows:

(1) Initialization:

At time $\mathrm{k}=1$, generate $\mathrm{N}$ particles $s_{0}^{i} \sim p\left(s_{0}\right) \quad i=0, \cdots, N$ and calculate the mean $\bar{s}_{k}^{i}$ and variance $P_{\mathrm{s}, 0}^{(i)}$.

(2) Sampling

For time $k=2,3, \cdots$, using unscented transmission with Gaussian mixture described in section $A$ to update the particles. Calculate the particle weights from the Eq. 35.

(3) Resampling

If Eq. 19 is satisfied, apply adaptive residual resampling from section B.

(4) Output:

Output the estimation result of state $s$, normalize the weights and calculate the variance for next time.

\section{Simulation Results}

In order to demonstrate the capability of the proposed GM-UPF with adaptive residual resampling algorithm, a great number of Monte Carlo simulations are carried out. The generic GPF and GM-UPF with adaptive residual resampling are realized respectively.
We evaluate the algorithm performance by estimation error and choose the root mean square error defined as RMSE:

$$
R M S E=\sqrt{\frac{1}{T} \sum_{k=1}^{T}\left(s_{k}-\tilde{s}\right)^{2}}
$$

Where $\mathrm{T}$ is the number of time step, $S_{k}$ is the real value of the target and $\tilde{s}_{k}$ is the estimation value.

The target dynamic state model and measurement model are described in section II. In measurement model, two static sensors are fixed at $(0,2000)$ and $(3000,1500)$. The target initial location is $(0,0)$ and the initial velocity is $(10,150)$. Set total time step endk $=50$, particle number $N_{s}=10$, measurement covariance $R=(10,4,10,4)$, and initial covariance $P_{-} 0=(1000,100,1000,100)$. For unscented transformation, the initial parameters assignment are $n a=4, k a p p a=0$, alpha $=1$ and beta $=2$.

The true position and the estimation by implementing GPF and GM-UPF are shown in Fig. 1. It can be seen that in random surfer dynamic model the estimated states of GMUPF are almost coincident with the true track, while the GPF estimations have some deviation.

Fig. 2 and Fig. 3 shows the estimation of $\mathrm{x}$ and $\mathrm{y}$, respectively. It can be also seen that GM-UPF outperforms compared with GPF.

The tracking state RMSE of GPF and GM-UPF can be seen from Fig. 4, which is applied to compare the accuracy of the estimation and demonstrate the consistency and convergence of the algorithms. It is obvious that the RMSE of GM-UPF is lower than GPF algorithm.

Here we give some simulation results of the effective particle size $\hat{N}_{\text {eff }}$, as shown in Fig. 5. It can obtain that $\left.\hat{N}_{\text {eff }}\right|_{G M-U P F}$ is more than $\left.\hat{N}_{\text {eff }}\right|_{G P F}$ noticeably. The results prove that GM-UPF prevails GPF in solving the particle degeneracy phenomenon.

\begin{tabular}{ccc} 
TABLE I. MEAN RESULTS ON 20 INDEPENDENT MONTE CARLOS \\
\hline \hline Variables & $\begin{array}{c}\text { GPF } \\
\text { (mean) }\end{array}$ & $\begin{array}{c}\text { GM-UPF } \\
\text { (mean) }\end{array}$ \\
\hline Time(s) & 0.1965 & 0.2320 \\
Xrmse & 20.7934 & 5.4786 \\
Yrmse & 35.514 & 7.8878 \\
$\hat{N}_{\text {eff }}$ & 4.9556 & 9.3937 \\
\hline \hline
\end{tabular}



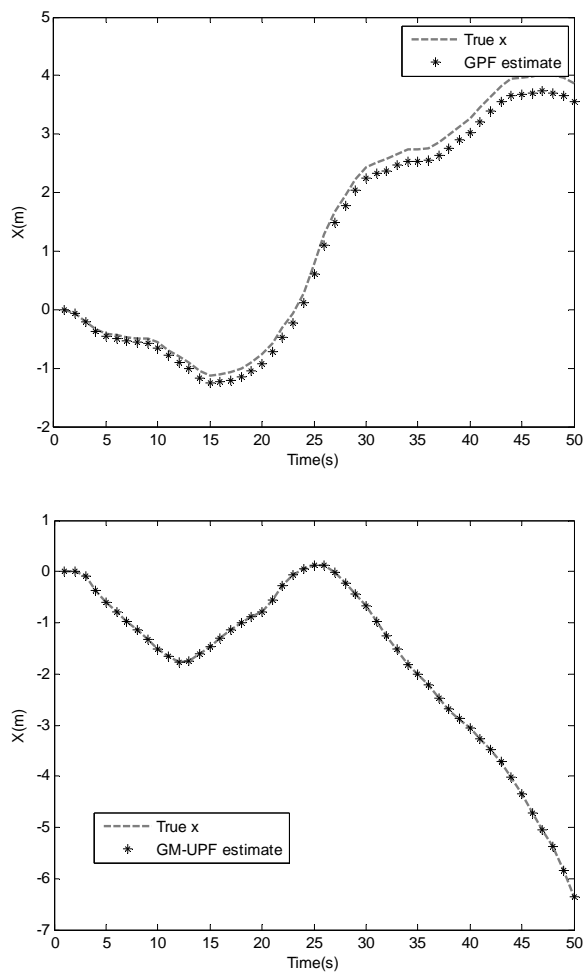

Figure 2. True $\mathrm{x}$ and the estimates of GPF and GM-UPF
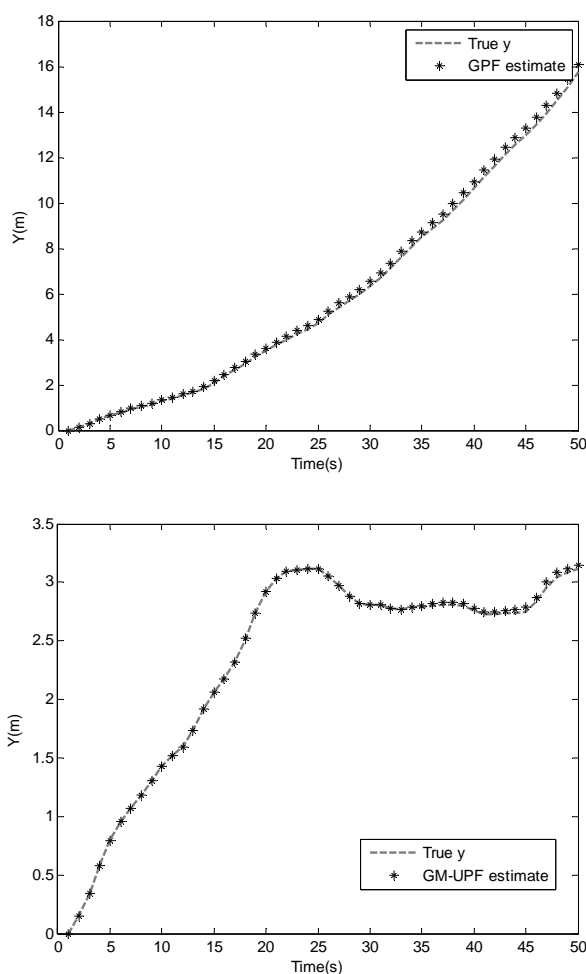

Figure 3. True y and the estimates of GM-UPF

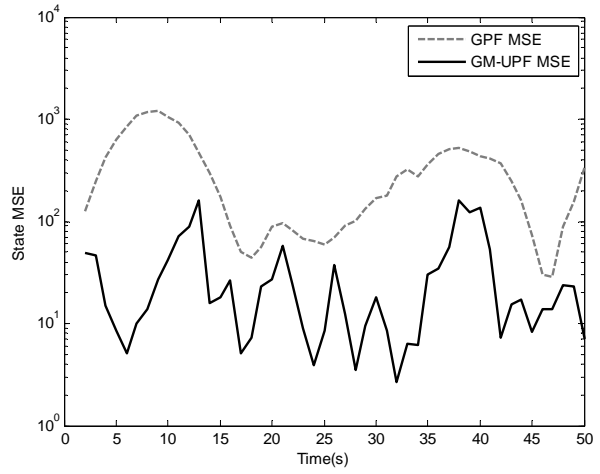

Figure 4. Track state RMSE error of GPF and GM-UPF

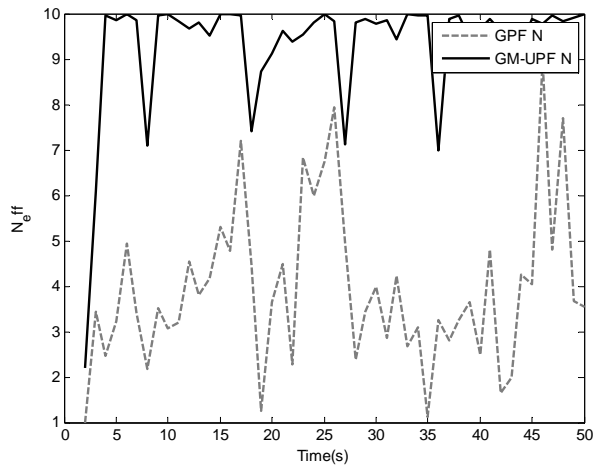

Figure 5. Effective particle size of GPF and GM-UPF

For further illustration, we run 20 independent Monte Carlos to compute mean results of variables shown in Table. 1. The elapsed time of GM-UPF is slightly longer than that of GPF in Table. 1, therefore the optimization can be made in the future work. From the results of Xrmse, Yrmse and $\hat{N}_{\text {eff }}$, we can see that the proposed GM-UPF is more outstanding, which proves the GM-UPF enhance the accuracy of nonlinear tracking algorithm.

\section{CONCLUSIONS}

In this paper, Gaussian mixture unscented particle filter with adaptive residual resample algorithm is presented. The performance of particle filter heavily depends on the importance density. By introducing Gaussian mixture unscented transformation as the importance density, the proposed GM-UPF algorithm approximates the posterior PDF more effectively. To overcome the defects of the traditional resample algorithms, the adaptive residual resample algorithm is applied in GM-UPF, thus solving the degeneracy phenomenon and particle impoverishment problem to a degree. Simulations are made to evaluate the performance of the proposed algorithm, range and range rate are utilized as the measurements with two sensors, and the random surfer dynamic model is used as states. Simulation results prove the GM-UPF algorithm can improve the performance compared with GPF, and demonstrate the GM- 
UPF algorithm is quite promising in nonlinear non-Gaussian filtering problems.

\section{ACKNOWLEDGMENT}

This paper is supported by the Major Science and Technology Project Fund of China (Grant No. 2012AA120602). The authors would also like to appreciate the anonymous reviewers and the editors for their many helpful suggestions, which have greatly improved the presentation of this paper.

\section{REFERENCES}

[1] Chen Z. Bayesian filtering: from Kalman filters to particle filters, and beyond. Adaptive System Laboratory, McMaster University, Hamilton, ON, Canada, 2003.

[2] Arulampalam M S, Maskell S, Gordon N, et al. A tutorial on particle filters for online nonlinear/non-Gaussion Bayesian tracking. IEEE Transactions on Signal Processing, 2002, 50(2): 174-188.

[3] Wang Zhengjie, Zhao xiaoguang, Wang Zhengjie, et al. Unscented particle filter with systematic resampling localization algorithm based on RSS for mobile wireless sensor networks. 2012 8th International Conference on Mobile Ad-hoc and Sensor Networks: 169-176.

[4] Hongjuan Zhang, Sixian Qin, Jianwen Ma, et al. Using Residual Resampling and Sensitivity Analysis to Improve Particle Filter Data Assimilation Accuracy. IEEE Geoscience and Remote Sensing Letters, 2013, 10(6): 1402-1408.

[5] Bunch P, Godsill S. Particle Filtering with Progressive Gaussian Approximations to the Optimal Importance Density. 2013 5th IEEE International Workshop on Computational Advances in Multi-Sensor Adaptive Processing (CAMSAP): 360-363.
[6] Bei Cao, CaiWen Ma, ZhenTao Liu. A Fine Resampling Algorithm for General Particle Filters. 2011 4th International Congress on Image and Signal Processing: 2695-2699.

[7] Marko S, Djogatovic, Milorad J, et al. A variable neighborhood search particle filter for bearings-only target tracking. Computers \& Operations Research, 2014, 52(B): 192-202.

[8] Bei Cao, Caiwen Ma, Zhentao Liu. Particle Filter with Fine Resampling for Bearings-only Tracking. 2012 International Workshop on Information and Electronics Engineering (IWIEE): 3685-3690.

[9] Bourgeois, Brian S. Using Range and Range Rate for Relative Navigations. NAVAL RESEARCH LAB STENNIS SPACE CENTER MS MARINE GEOSCIENCES DIV, 2007.

[10] Bizup D.F, Brown D.E. Maneuver Detecion Using the Radar Range Rate Measurement. IEEE Transactions on Aerospace and Electronic Systems, 2004, 40(1): 330-336.

[11] Subash J. Unscented particle filter in road extraction from high resolution satellite images. International Conference on Recent Trends in Information Technology (ICRTIT), 2012, 123-129.

[12] Guoshen Yu, Morel, J -M. A fully affine invariant image comparison method. IEEE International Conference on Acoustics, Speech and Signal Processing, 2009, 1597-1600.

[13] Tiancheng Li, Tariq Pervez Sattar, Shudong Sun. Deterministic resampling Unbiased sampling to avoid sample impoverishment in particle filters. Signal Processing, 2012, 92(7):1637-1645.

[14] Lianmeng Jiao, Quan Pan, Yan Liang et al. A nonlinear tracking algorithm with range-rate measurements based on unbiased measurement conversion. 2012 15th international conference on information fusion, 1400-1405. 